\title{
Talimogene Laherparepvec
}

National Cancer Institute

\section{Source}

National Cancer Institute. Talimogene Laherparepvec. NCI Thesaurus. Code C61314.

An ICP34.5, ICP47-deleted, oncolytic herpes simplex type-1 virus (HSV-1) based on the JS1 strain, and encoding the immunostimulating factor human cytokine granulocytemacrophage colony stimulating factor (GM-CSF) with potential immunostimulating and antineoplastic activities. Upon intratumoral injection, talimog ene laherparepvec selectively infects and replicates in tumor cells, thereby inducing tumor cell lysis. In addition, GM-CSF attracts dendritic cells (DCs) and may stimulate a cytotoxic $T$ cell response against tumor cells, which results in immune-mediated tumor cell death. Deletion of the gene encoding for ICP34.5 provides tumor selectivity and prevents replication in healthy cells. As ICP47 blocks antigen presentation in HSV-infected cells, deletion of this gene may induce a more potent antitumor immune response in the tumor cells. Additionally, deletion of ICP47 causes increased expression of the HSV US11 gene and allows US11 to be expressed as an immediate early and not a late gene. This further enhances the degree of viral replication and oncolysis of tumor cells. 